\title{
Polarization of Dielectrics by Acceleration
}

\author{
L.A. Melnikovsky*
}

November 13, 2018

\begin{abstract}
We argue that acceleration induces electric polarization in usual dielectrics. Both accelerations in superfluid ( $\dot{\mathbf{v}}_{\mathrm{s}}$ and $\dot{\mathbf{v}}_{\mathrm{n}}$ ) participate in the medium polarization. Excitations contribution to the polarization is calculated at low temperatures. Estimates of the effect show order of magnitude agreement with recent experimental results on electric effect of superflow.
\end{abstract}

\section{Introduction}

Excitation of electric currents by metal acceleration is well known and usually associated with the Stewart-Tolman [1] effect. Similar phenomenon exists in dielectrics. In this paper we show that acceleration polarizes dielectrics. There are two formally separated mechanisms of the effect. Individual accelerated molecules acquire intrinsic dipole moment. This contribution is discussed in Sec 2 Collective polarization caused by the medium inhomogeneity required for the accelerated motion is considered in Sec 3

As reported [2] by A.S. Rybalko, a second-sound wave gives rise to an electric field in superfluid.* In Sec廿 we show that electric effect of superflow can be interpreted as a manifestation of the acceleration-induced polarization. Eventually in Sec 5 we estimate the effect magnitude in the second-sound wave.

\section{Gravitoelectric effect}

Applied uniform electric field $\mathbf{E}$ polarizes isotropic dielectric, in linear approximation its polarization (dipole moment per unit volume) is given [5] by the equation

$$
\mathbf{P}=n \mathbf{d}=\varkappa \mathbf{E},
$$

*E-mail: leva@kapitza.ras.ru

* Subsequent work 3 was aimed to discover electric effect of the superfluid flow in torsional oscillator. There has also been an attempt to describe these experimental results phenomenologically [4. 
where $\mathbf{d}$ is a single atom dipole moment, $n$ is the atom density, $\varkappa=(\varepsilon-1) / 4 \pi$ is the electric susceptibility, and $\varepsilon$ is the permittivity of the medium. In presence of an external gravity field $\mathrm{g}$ equation (1) should be extended to include additional term

$$
n \mathbf{d}=\varkappa \mathbf{E}+\mathbf{P}_{g}=\varkappa \mathbf{E}+\gamma \mathbf{g}
$$

Phenomenologically we can not calculate the "gravitoelectric susceptibility" $\gamma$, it is an intrinsic property of the dielectric. We can, however, estimate it from microscopic analysis. Consider the dipole moment $\mathbf{d}$ of a single atom. Both electric and gravity forces acting upon it urge to tear electric cloud away from the nucleus. The polarization must therefore be equal to zero if these two effects compensate each other. Electric "tear force" (the difference between the forces acting on the nucleus and on the electrons) is approximately $F_{e} \sim 2 E Z e$, where $Z$ is the atom number and $e$ is the elementary charge. Gravity effectively acts on the nucleus only, appropriate "force" is $F_{g} \sim g M$, where $M$ is the atom mass. Equating these two "forces" we obtain $\gamma \sim \varkappa M /(2 Z e)$.

So far we considered the dielectric at rest. Influence of the velocity, unless it is comparable to the speed of light, can be neglected, but equivalence principle of the General Relativity dictates how the acceleration $\dot{\mathbf{v}}$ enters (2)

$$
\mathbf{P}_{g}=\gamma(\mathbf{g}-\dot{\mathbf{v}})
$$

\section{$3 \quad$ Flexoelectric effect}

Uniform medium polarization is a single atom dipole moment times the atom density (1). In a nonuniform medium (and even uniform gravity field breaks the system homogeneity) higher order electric moments (quadrupole etc.) also contribute to the macroscopic polarization $\mathbf{P}=n \mathbf{d}+\mathbf{P}_{f}$. These contributions should be separable since they depend differently on external field and its gradients 6]. Similar phenomenon is known, e.g., in liquid crystals and is referred to as quadrupole flexoelectric effect ${ }^{\dagger}$ [7.

To find the quadrupole contribution to the polarization we follow the arguments used in Ref. 5 to introduce the polarization itself. If the medium has no external charges nor the atoms posses any intrinsic dipole moment, then the total dipole moment of a body of any shape is identically zero

$$
\int \mathbf{P}_{f} \mathrm{~d} V=0 .
$$

Consequently, the polarization can be expressed as a divergence of some tensor

$$
P_{f}^{i}=-\frac{1}{6} \frac{\partial Q^{i j}}{\partial x^{j}}
$$

${ }^{\dagger}$ Possible manifestation of the effect in superfluid ${ }^{3} \mathrm{He}$ vortices is discussed in Ref. 8 . 
and $Q^{i j}$ is zero outside the body. To elucidate the meaning of $Q^{i j}$ consider the total "generalized" quadrupole moment" of the body $\mathfrak{Q}^{i j}$

$$
\mathfrak{Q}^{i j}=3 \int x^{i} x^{j} \varrho \mathrm{d} V=-3 \int x^{i} x^{j} \frac{\partial P_{f}^{k}}{\partial x^{k}} \mathrm{~d} V=\int Q^{i j} \mathrm{~d} V .
$$

Here $\varrho=-\nabla \mathbf{P}_{f}$ is the charge density and $Q^{i k}$ is assumed to be symmetric without loss of generality. Tensor $Q^{i j}$ is therefore a "generalized quadrupole moment" density per unit volume.

Macroscopic electric field $\mathbf{E}=\mathbf{D}-4 \pi \mathbf{P}$ is the average microscopic field $\mathbf{E}=\langle\mathbf{e}\rangle$ and can be detected within the medium by a probe charge. To find the field outside the medium, the Maxwell equations should be equipped with the boundary conditions. They should be corrected if bulk quadrupole moment is taken into account. Appropriate contribution to the contact potential for the interface between the medium and vacuum (denoted below by $m$ and $v$ respectively) is determined by the double layer at the medium surface. To find it consider the integral along an infinitesimal path $m \rightarrow v$ crossing the surface:

$$
\varphi_{v}-\varphi_{m}=-\int_{m}^{v} \mathbf{E} \mathrm{d} \mathbf{s}=4 \pi \int_{m}^{v} P_{f}^{i} \mathrm{~d} x^{i}=-\frac{2 \pi}{3} \int_{m}^{v} \frac{\partial Q^{i j}}{\partial x^{j}} \mathrm{~d} x^{i}=\frac{2 \pi}{3} Q^{i j} n^{j} n^{i} .
$$

Here $\mathbf{n}$ is a unit vector normal to the surface and directed outwards the medium.

For an isotropic medium (normal liquid) $Q^{i k}=Q \delta^{i k}$ and can be estimated as $Q \sim-Z e r_{0}^{2} \rho / M$, where $r_{0}$ is the atom radius and $\rho$ is the liquid density. This gives for the polarization

$$
\mathbf{P}_{f} \sim \frac{Z e}{6} \nabla\left(\frac{M^{2 / 3}}{4 \rho^{2 / 3}} \frac{\rho}{M}\right) \sim \phi \nabla\left(\rho^{1 / 3}\right)
$$

where $\phi=Z e /\left(24 M^{1 / 3}\right)$.

\section{Polarization of Superfluid}

Superfluid may posses two types of motion: superfluid and normal flows characterized by the velocities $\mathbf{v}_{\mathrm{s}}$ and $\mathbf{v}_{\mathrm{n}}$, and by the densities $\rho_{\mathrm{s}}$ and $\rho_{\mathrm{n}}$. Equations (3) and (45) therefore require generalization to this two-fluid case.

Phonons, the most important excitations at low temperatures, can be described by macroscopic hydrodynamics. [10] $]^{\S}$ Equation (3) when averaged over thermal phonons gives macroscopic gravitoelectric polarization as $\mathbf{P}_{g}=-\langle\gamma \dot{\mathbf{v}}\rangle$. Microscopic acceleration is obtained from the Euler equation

$$
\dot{\mathbf{v}} \equiv \frac{\mathrm{d} \mathbf{v}}{\mathrm{d} t} \equiv \frac{\partial \mathbf{v}}{\partial t}+v^{i} \frac{\partial \mathbf{v}}{\partial x^{i}}=-\frac{\nabla \mathfrak{p}}{\rho}
$$

\footnotetext{
${ }^{\ddagger}$ Conventional quadrupole moment tensor is traceless.

§Similar approach is used in Ref. 9] to calculate optical anisotropy in superfluid.
} 
where $\mathfrak{p}(\rho)$ is the microscopic pressure. Electric susceptibility of helium is very small and is therefore proportional to the density. This also applies to the gravitoelectric susceptibility $\gamma \propto \varkappa \propto \rho$. From (6) we get

$$
\mathbf{P}_{g}=\frac{\gamma}{\rho}\langle\nabla \mathfrak{p}\rangle=\frac{\gamma}{\rho} \frac{\partial}{\partial x^{i}}\left\langle\Pi^{i k}-\rho v^{i} v^{k}\right\rangle=\frac{\gamma}{\rho} \frac{\partial}{\partial x^{i}}\left(\Pi^{i k}-\left\langle\rho v^{i} v^{k}\right\rangle\right),
$$

where $\Pi^{i k}$ is the momentum flux density tensor. Its microscopic and average macroscopic values are given by

$$
\Pi^{i k}=\mathfrak{p} \delta^{i k}+\rho v^{i} v^{k} \quad \text { and } \quad \Pi^{i k}=v_{\mathrm{s}}^{i} j^{k}+v_{\mathrm{n}}^{k} j^{i}-\rho v_{\mathrm{n}}^{k} v_{\mathrm{s}}^{i}+p \delta_{i k}
$$

respectively, where $\mathbf{j}$ is the mass flux and $p$ is the pressure. Anisotropic part of $\left\langle\rho v^{i} v^{k}\right\rangle$ is quadratic with respect to the relative velocity $\mathbf{w}=\mathbf{v}_{\mathbf{n}}-\mathbf{v}_{\mathbf{s}}$, in linear approximation it can be neglected $\left\langle\rho v^{i} v^{k}\right\rangle=\delta^{i k}\left\langle\rho v^{2}\right\rangle / 3$. In phonons mean kinetic energy $E_{k}=\left\langle\rho v^{2} / 2\right\rangle$ is equal to mean potential energy

$$
E_{p}=\frac{1}{2 \rho} \frac{\partial p}{\partial \rho}\left\langle(\rho-\langle\rho\rangle)^{2}\right\rangle=\frac{c^{2}}{2 \rho}\left\langle(\rho-\langle\rho\rangle)^{2}\right\rangle,
$$

where $c$ is the velocity of sound". In other words $\left\langle\rho v^{2}\right\rangle=\delta E_{0}$, where $\delta$ designates the increment with respect to the value at $T=0$ (neglecting the ground state energy), i.e., $\delta E_{0}=E_{0}-\left.E_{0}\right|_{T=0}$. Here $E_{0}=T \sigma \rho+\mu \rho-p$ is the energy density in the frame of reference of the superfluid component, $\sigma$ and $\mu$ are the entropy and the chemical potential per unit mass.

$$
\mathrm{d} E_{0}=T \mathrm{~d}(\rho \sigma)+\mu \mathrm{d} \rho=T \rho \mathrm{d} \sigma+\frac{p+E_{0}}{\rho} \mathrm{d} \rho=C \rho \mathrm{d} T+\frac{W}{c^{2}} \mathrm{~d} p,
$$

where $W=\left(p+E_{0}\right) / \rho$ is the heat function per unit mass. Finally combining (7), (8), and (10) we get

$$
\mathbf{P}_{g}=\frac{\gamma}{\rho}\left(1-\frac{\delta W}{3 c^{2}}\right) \nabla p-\frac{\gamma C}{3} \nabla T=\frac{\gamma}{\rho}\left(1-\frac{\delta W}{3 c^{2}}-\frac{C}{3 \sigma}\right) \nabla p+\frac{\gamma C}{3 \sigma} \nabla \mu .
$$

This can also be expressed with the help of linearized superfluid hydrodynamics equations as

$$
\mathbf{P}_{g}=\frac{\gamma C \rho_{\mathrm{n}}}{3 \rho \sigma} \dot{\mathbf{w}}-\frac{\gamma}{\rho}\left(1-\frac{\delta W}{3 c^{2}}\right)\left(\rho_{\mathrm{s}} \dot{\mathbf{v}}_{\mathrm{s}}+\rho_{\mathrm{n}} \dot{\mathbf{v}}_{\mathrm{n}}\right)=-\gamma_{\mathrm{n}} \dot{\mathbf{v}}_{\mathrm{n}}-\gamma_{\mathrm{s}} \dot{\mathbf{v}}_{\mathrm{s}}
$$

where

$$
\gamma_{\mathrm{n}}=\frac{\gamma \rho_{\mathrm{n}}}{\rho}\left(1-\frac{\delta W}{3 c^{2}}-\frac{C}{3 \sigma}\right), \quad \gamma_{\mathrm{s}}=\frac{\gamma}{\rho}\left(\rho_{\mathrm{s}}-\frac{\rho_{\mathrm{s}} \delta W}{3 c^{2}}+\frac{C \rho_{\mathrm{n}}}{3 \sigma}\right) .
$$

IWe use the same symbols for the microscopic and the average values of such quantities as density $\rho$ and momentum flux $\Pi^{i k}$, while keep $\mathfrak{p}$ and $p$ distinguishable since $\langle\mathfrak{p}\rangle \neq p$.

$\|$ No distinction is made between isothermal and adiabatic compressibility $\partial p / \partial \rho=c^{2}$ nor between heat capacity $C$ at constant pressure and density. 
Similarly, averaged (5) reads

$$
\mathbf{P}_{f} \sim \phi \nabla\left\langle\rho^{1 / 3}\right\rangle=\phi \nabla\left(\rho^{1 / 3}-\frac{1}{9 \rho^{5 / 3}}\left\langle(\rho-\langle\rho\rangle)^{2}\right\rangle\right) .
$$

Employing (9) we get for the phonons

$$
\mathbf{P}_{f} \sim \phi \nabla\left(\rho^{1 / 3}-\frac{1}{9 c^{2} \rho^{2 / 3}} \delta E_{0}\right)=\phi \nabla\left(\rho^{1 / 3}-\frac{\rho_{\mathrm{n}}}{12 \rho^{2 / 3}}\right),
$$

using $\rho_{\mathrm{n}}=4 \delta E_{0} /\left(3 c^{2}\right)$ for the phonon term in the normal density [11.

Rotons, unlike phonons, being the short-wavelength excitations, are not described by macroscopic hydrodynamics. Their contribution to the quadrupole moment is not necessarily scalar, i.e., $\mathcal{Q}^{i j}=Q^{i j}-Q^{k k} \delta^{i j} / 3 \not \equiv 0$. Contribution of a single roton to $\mathcal{Q}^{i j}$ can be estimated (see (14)

$$
q^{i j} \sim \frac{\phi \epsilon}{c^{2} \rho^{2 / 3}}\left(\frac{p^{i} p^{j}}{p^{2}}-\frac{\delta^{i j}}{3}\right),
$$

where $\mathbf{p}$ and $\epsilon$ are the roton momentum and energy. Total quadrupole moment density is therefore

$$
\mathcal{Q}^{i j}=\int q^{i j} n(\epsilon-\mathbf{p w}) \frac{\mathrm{d} \mathbf{p}}{(2 \pi \hbar)^{3}} \sim \frac{\phi \rho_{\mathrm{n}} \Delta}{T \rho^{2 / 3}}\left(\frac{w^{i} w^{j}}{5 c^{2}}-\frac{\delta^{i j} w^{2}}{15 c^{2}}\right),
$$

where $\epsilon \approx \Delta$ is the roton energy gap. Here we assumed Boltzmann distribution $n(\epsilon)$ for rotons and by $\rho_{\mathrm{n}}$ imply purely roton contribution to the normal density [11] $\rho_{\mathrm{n}}=\int n(\epsilon) p^{2} \mathrm{~d} \mathbf{p} /\left(24 \pi^{3} \hbar^{3} T\right)$. Equation (15) implies nontrivial orientation dependence of the contact potential.

\section{Discussion}

Recent experiments [2] demonstrate that a second-sound wave gives rise to an electric field in superfluid. We can estimate the expected effect in these experiments from the equations (11) and (15).** Neither pressure nor total density gradients are present in a second sound wave and the relative velocity amplitude $w^{\prime}$ can be obtained from the temperature amplitude $T^{\prime}$ as $w^{\prime}=S T^{\prime} /\left(c_{2} \rho_{\mathrm{n}}\right)$, where $c_{2}$ is the second sound velocity.

The gravitoelectric contribution to the voltage across a second sound wavelength is then

$$
U_{g}=-\int \mathbf{E} \mathrm{d} \mathbf{x} \sim \int 4 \pi P_{g} \mathrm{~d} x \sim-4 \pi \frac{\gamma}{3} C T^{\prime} \sim-\frac{(\varepsilon-1) M}{12 e} C T^{\prime},
$$

while the contact potential contribution is given by

$$
U_{c} \sim \frac{\pi}{180} \frac{Z e \rho^{1 / 3}}{M^{1 / 3}} \frac{S^{2} T^{\prime 2}}{\rho \rho_{\mathrm{n}} c^{2} c_{2}^{2}} \frac{\Delta}{T} .
$$

**Scalar quadrupole contribution (as described by Eq 14) is unobservable with external probes: contact potential cancels the field outside of the liquid. 
The later is quadratic with respect to the relative velocity (and therefore to the temperature amplitude) and oscillates at the double frequency.

\section{Acknowledgements}

I am grateful to A.F. Andreev, K.O. Keshishev, V.I. Marchenko, and G.E. Volovik for fruitful discussions. I would also like to thank University of New Mexico for its hospitality. This work was supported in parts by RFBR grants 06-02-17369, 06-02-17281 and RF president program 7018.2006.2.

\section{References}

[1] R.C. Tolman, T.D. Stewart, Phys. Rev. 8, 97 (1916).

[2] A.S. Rybalko, Low Temperature Physics 30, Issue 12, 994 (2004).

[3] A.S. Rybalko, S.P. Rubets, Low Temperature Physics 31, Issue 12, 623 (2005).

[4] A.M. Kosevich, Low Temperature Physics 31, Issue 1, 37 (2005).

[5] L.D. Landau, E.M. Lifshitz, Electrodynamics of Continuous Media (Pergamon Press, Oxford, 1984).

[6] Ya.I. Frenkel, Electrodynamics, §5 (ONTI, Moscow, 1934, in Russian).

[7] J. Prost, J.P. Marcerou, J. Phys. 38, 315 (1977).

[8] G.E. Volovik, JETP Letters 39, Issue 4, 200 (1984).

[9] A.F. Andreev, JETP Letters 31, Issue 3, 175 (1980).

[10] L.D. Landau, E.M. Lifshitz, Fluid Mechanics (Pergamon Press, Oxford, 1987).

[11] E.M. Lifshitz, L.P. Pitaevskii, Statistical Physics, Part 2 (Pergamon Press, Oxford, 1981). 